\title{
Double primary cancers registered in a tertiary care hospital: review of two cases
}

\author{
Amrallah A. Mohammad¹,2, Abdullah S. Al-Zahrani , Hani M.EL-Khatib \\ 'Oncology Center, King Abdullah Medical City - Holy Capital, Saudi Arabia.
}

${ }^{2}$ Department of Medical Oncology,

Faculty of Medicine, Zagazig University, Egypt

\begin{abstract}
In our centre, among 1965 registered cancer patients between May 2011 and December 2013, we report three cases with multiple primary malignant neoplasms. One of them was excluded due to lack of data, and so we present the remaining two cases. The first case is an 82-year-old female patient with colon and thyroid cancer and the second case is a 61-year-old female patient with colon and breast cancer. Both cases were metachronous and discovered accidently during the regular follow up, and managed with a curative intent. Conclusion: It is important for the clinicians to keep in mind that individuals with cancer are at increased risk for subsequent primary malignancies, which must be differentiated from recurrent or metastatic disease.
\end{abstract}

Keywords: Synchronous/metachronous • Primary malignancy • Multiple primary malignancies

(C) De Gruyter Open

\section{Introduction}

Multiple primary malignant neoplasms (MPNMs) are defined as two or more malignancies in a single individual without any relationship between cancers [1]. It is not very rare, and has a marked increase in incidence in the past few years, possibly explained by the greater awareness, improved diagnostic techniques and facilities [2-5]. This entity was first described by Billroth at the end of the 19th century [6]. One of the earliest statistical analyses of MPMNs was carried out by Bugher in 1934 [7]. In our report, we present two cases with double primary tumours; one is a patient with colon cancer and a metachronous thyroid cancer and the other is a patient with colon cancer and a metachronous breast cancer.

\section{Case Report (1)}

An 82-year-old female patient was referred to our centre (King Abdullah Medical Center; Holly Capital) as a case of acute abdomen on 8 December 2012 for further investigation and management.
Her condition had started 2 months before with constipation of gradual onset and progressive course associated with nausea and vomiting, which finally resulted in a complete obstruction.

She had a history of diabetes mellitus type II and was on oral hypoglycaemic medications; she was subjected to an open cholecystectomy procedure more than 10 years ago due to gall stones. She had no relevant family history. On physical examination, her abdomen was distended and tender on palpation. There was a palpable mass in the left iliac region, and bowel sounds were not audible. On laboratory testing, she had a serum chemistry panel and complete blood count within normal limits. Computed tomography scan of the upper and lower abdomen revealed a colorectal mass causing complete intestinal obstruction (ileus). Urgent laparotomy was performed with resection of the colonic mass and diverting colostomy. The histology examination revealed (Figure 1):

Moderately differentiated adenocarcinoma

- Tumour size $3 \mathrm{~cm}$

- Tumour invades through the muscularis propria into the pericolic fat 


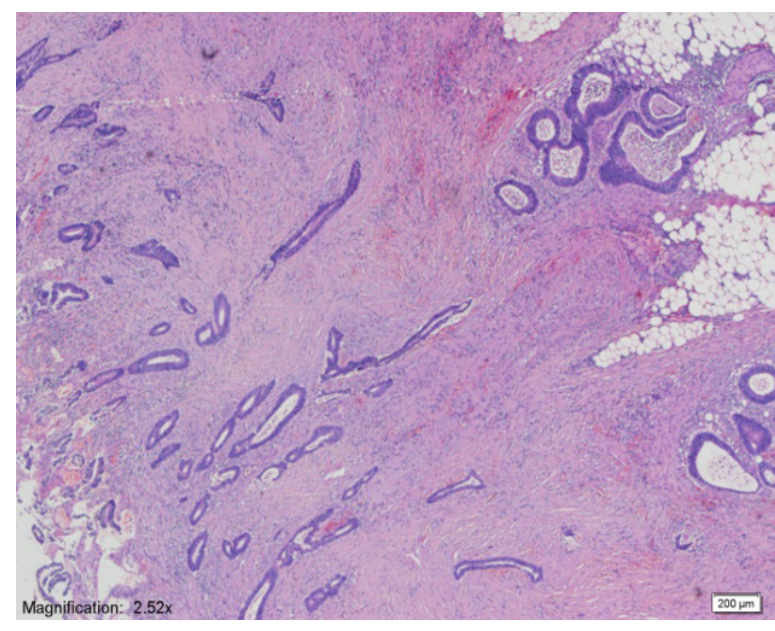

Figure 1. Adenocarcinoma of recto sigmoid, magnification $2.52 \times$ $H \& E$.

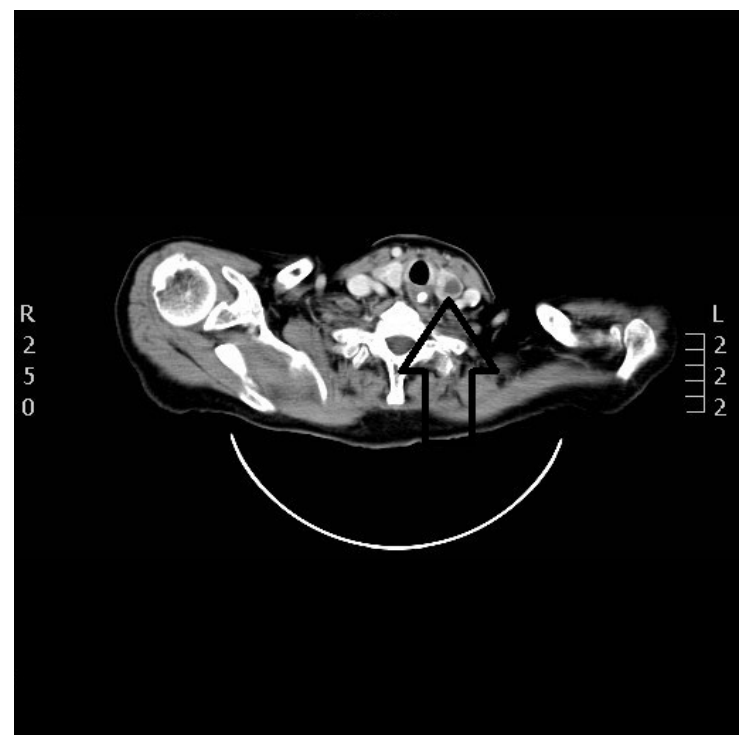

Figure 2. Post-contrast computed tomography showed left thyroid nodule.

- $\quad$ One lymph node was positive out of seven resected

- Lymphovascular invasion: not identified

- Perineural invasion: not identified.

The patient had a smooth recovery and was discharged 10 days after the operation. Post-operation evaluation and staging by computed tomography scan of the chest, abdomen and pelvis revealed no evidence of tumour residual or distant spread; at this time, she was started on capecitabine as adjuvant treatment and completed eight cycles without any significant adverse events. Three months later, computed tomography scan of the chest, abdomen and pelvis as part of her follow up revealed a left thyroid nodule measuring $2.6 \mathrm{~cm} \times$ $1.2 \mathrm{~cm}$ with multiples cystic areas inside (Figure 2).

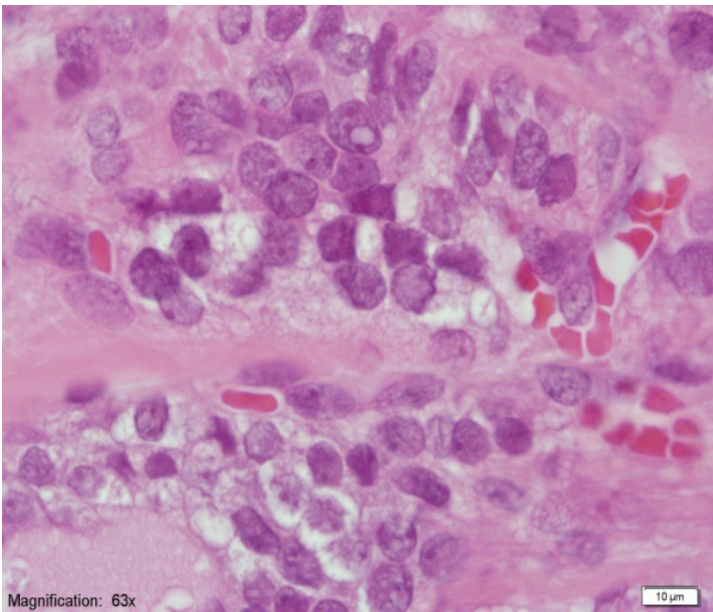

Figure 3. Thyroid carcinoma, nuclear grooves and inclusion, magnification $63 \times, H$ \&

Fine needle aspiration cytology of the nodule revealed papillary carcinoma; at this time, she was transferred to the Head and Neck clinic for further management. Total thyroidectomy was performed and the histology examination revealed (Figure 3 ) the following:

- Left thyroid lobe: papillary carcinoma, follicular variant with negative surgical margins and no lymphovascular invasion

- $\quad$ Right thyroid lobe and isthmus: multinodular goiter.

Whole body iodine scan showed residual functioning thyroid tissue in the right lobe bed and regional lymph nodes without evidence of distant metastasis. She subsequently received an ablative dose of radioactive iodine 131 with $30 \mathrm{mCi}$. Currently, she is under follow up in the outpatient clinic with L-thyroxin therapy and calcium tablet supplementation.

\section{Case Report (2)}

A 61-year-old female colon cancer patient was transferred to our oncology centre in November 2011 for further management. Her condition had started 2 months before with intermittent diarrhoea, alternating with rectal bleeding, and associated with abdominal pain and weight loss. The patient was referred as an emergency to the nearest hospital with a possible situation of obstructive ileus, with vomiting and abdominal distension. Clinical examination revealed a distended, tender abdomen and inaudible bowel sounds.

Computed tomography scan of the upper and lower abdomen revealed a malignant stricture in the sigmoid colon with proximal colonic dilatation. In routine blood tests no abnormal findings were revealed. Urgent 


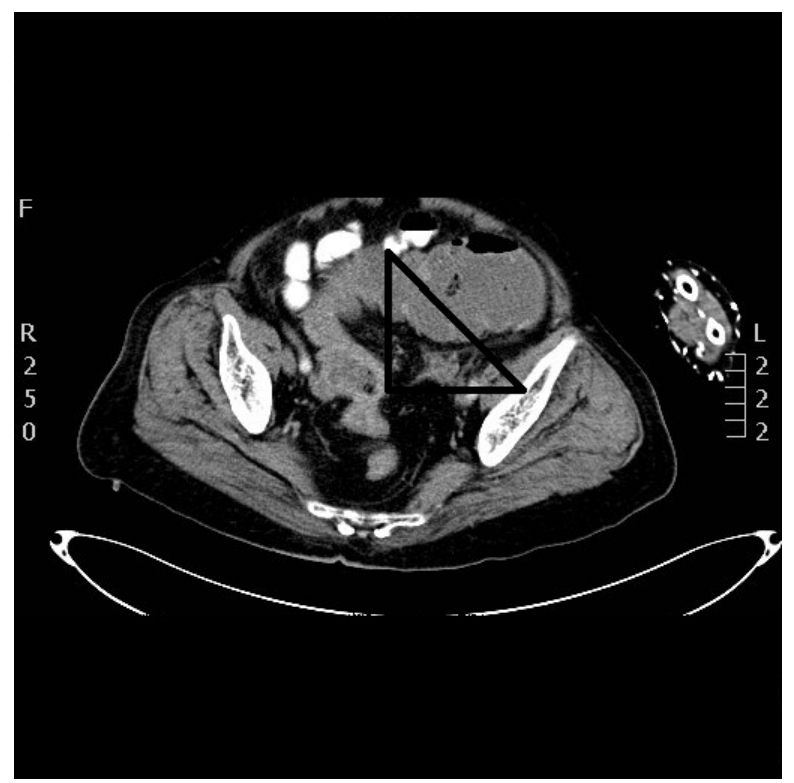

Figure 4. Post-contrast computed tomography abdomen revealed malignant stricture sigmoid colon with proximal colonic dilatation

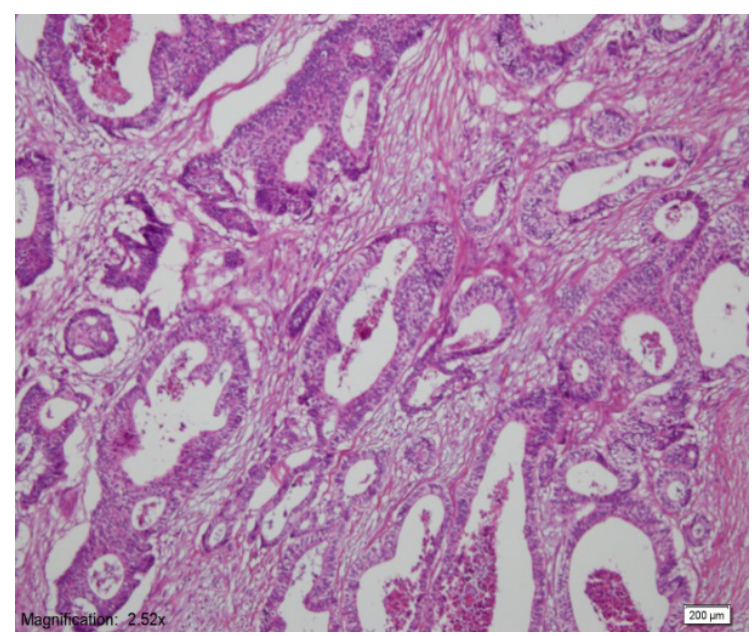

Figure 5. Adenocarcinoma of colon, magnification $2.52 \times, \mathrm{H}$ \& E.

laparotomy had to be performed with right hemicolectomy and end-to-end anastomosis. The histology examination revealed adenocarcinoma of the colon (Figure 4).

The computed tomography of the abdomen revealing the relevant mass is shown in Figure 5 . The histopathology report showed:

- Moderately differentiated adenocarcinoma infiltrating the entire thickness of the wall and adjacent pericolic fat

- Presence of perivacular and perineural invasion

- Negative surgical margins

- $\quad$ Three out six lymph nodes positive for metastasis.

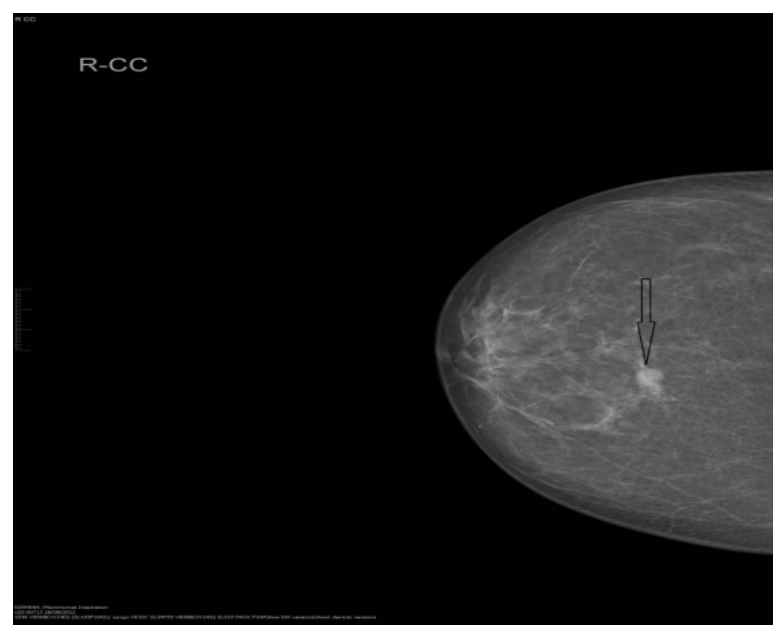

Figure 6. Mammogram revealed ill-defined speculated soft tissue mass.

Post-operative re-assessment showed no local recurrence or metastatic spread. Being a stage III colon cancer patient, she subsequently received adjuvant chemotherapy with six cycles of FOLFOX. Since the completion of adjuvant chemotherapy, she was under regular follow up in our oncology centre. Six months after the end of adjuvant chemotherapy, a small right breast mass $(2 \mathrm{~cm} \times 2 \mathrm{~cm}$ ) was clinically discovered accidently. Bilateral mammogram and complementary breast ultrasonography revealed a small right breast mass $(1.5 \mathrm{~cm} \times 1.5 \mathrm{~cm})$ with microclacification, which was highly suspicious for malignancy (Figure 6).

Right breast lumpectomy with right axillary sentinel lymph node dissection was performed and the histology revealed the following (Figure 7):

- Invasive ductal carcinoma

- Grade II

- $\quad$ Tumour size $1.0 \mathrm{~cm} \times 0.6 \mathrm{~cm}$

- Negative surgical margins

- Negative lymphovacular and perineural invasion

- $\quad$ All lymph nodes were free of disease $(0 / 5)$

- Estrogen Receptor (ER) and Progesterone Receptor (PR) were positive and (human epidermal growth factor receptor 2) HER-2 was negative (score 0).

The patient was diagnosed as having breast cancer stage I (intermediate risk) and subsequently received four cycles of adjuvant chemotherapy with anthracycline and cyclophosphamide, followed by adjuvant radiotherapy and adjuvant hormonal therapy. Thereafter, she continued to be under regular follow up in the outpatient clinic of our oncology centre. 


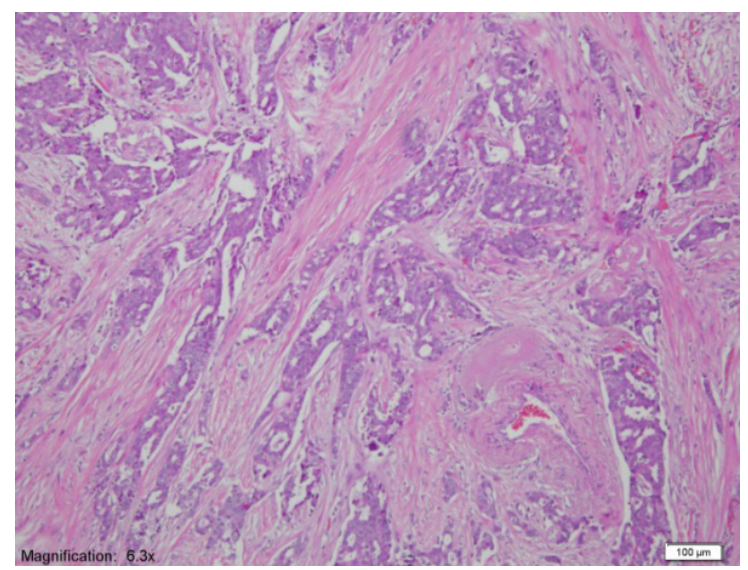

Figure 7. Invasive ductal carcinoma of breast, magnification $6.3 \times$, $H \& E$.

\section{Discussion}

MPMNs may be synchronous or metachronous neoplasias, depending on the interval between the respective diagnosis. Synchronous malignancies are second neoplasias occurring simultaneously or within 6 months after the first malignancy, while metachronous multiple malignancies are secondary neoplasias developed more than 6 months after from the first malignancy [8].

Three diagnostic criteria have been proposed for MPMNs: 1) each tumour must present definite features of malignancy, 2) each must be distinct and 3) the chance of one being a metastasis of the other must be excluded [9].

MPMNs are classified into four types: 1) multicentric, if the two distinct carcinoma arise in the same organ or tissue; 2) systemic, if they arise on anatomically or functionally allied organs of the same system; 3) paired organs, as in the breasts; and 4) random, if they occur as a co-incidental or accidental association in unrelated sites [10].

The frequency rate ranges between $0.7 \%$ and $14.5 \%$ $[11,12]$. This wide range is misleading and questionable as no acceptable average rate can be quoted. There are a number of factors responsible for this wide range including the following:

- The majority of published data on MPMNs are case reports that are difficult to assemble into a meaningful frequency rate $[13,14]$.

- It is not clear in many of the reports whether the mentioned ranges refer to the number of patients with MPMNs as a whole, or to synchronous, metachronous or both $[15,16]$.
- Most reports lack unified definition of MPMNs, synchronous and metachronous lesions [17, 18].

Probable aetiology and mechanisms of multiple primary cancers include genes, environmental factors, treatment effects or combinations of the above mentioned. When researchers focus on genes as causal mechanisms, they often concentrate on cases diagnosed earlier in life in young individuals [19].

Although, for example, bladder and prostate cancer can coexist in the same individual rather frequently [2022], the combination of colon cancer and either thyroid cancer or breast cancer are uncommon. In the two cases described here, there was no apparent family history and no predisposing factors or either malignancy.

The prognosis of patients with MPMs can be determined independently by the stage of each malignancy. Surgery is the treatment of choice for metachronous multiple primary malignancies if curative resection of each malignant tumour can be performed [23]. In the cases presented here, metachronous malignancies were discovered accidentally during regular follow up. In both cases, they were in early stages that gave us the chance for curative treatment. A large population base is needed in order to perform meaningful research on multiple primary cancers; such a population base will require extensive collaboration between cancer registries.

\section{Conclusion}

A cancer diagnosis should not exclude the existence of another concomitant malignancy, especially in patients older than 60 years of age.

\section{Consent}

Written informed consent was obtained from the patients for publication of this case report and any accompanying images. A copy of the written consent is available for review from the Editor-in-Chief of this journal.

\section{Conflict of Interest}

The authors certify that there is no actual or potential conflict of interest in relation with this article. 
[1] Yhim HY, Kim HS, Lee NR, Kwak JY, Yim CY. (2010) Quadruple primary malignancies of liver, bladder, lung and stomach in one patient. Tumori 96: 787-791.

[2] Vaslamatzis M, Alevizopoulos N, Petraki C. Second primary neoplasms (SPN) in cancer patients. Proc ASCO2003; 22: 3581.

[3] Morgenfeld EL, Tognelli F, Gil Deza E. Synchronous and metachronous second (ST) and third (TT) primary tumors (PT) in a large patient population. Proc ASCO 2003; 22: 3152.

[4] Kaneko S, Yamaguchi N. Epidemiological analysis of site relationships of synchronous and metachronous multiple primary cancers in the National Cancer Center, Japon, 1962- 1996. Jpn J Clin Oncol1999; 29: 96-105.

[5] Aydiner A, Karadeniz A, Uygun K. Multiple primary neoplasms at a single institution: differences between synchronous and metachronous neoplasms. Am J Clin Oncol 2000; 23: 364-370.

[6] Billroth T. Die allgemeine chirurgische pathologie und therapie in 51 vorlesungen. In: Reimer G, editor. Handbuch für studierende und ärzte. Berlin; 1882. p. 908.

[7] Bugher JC. The probability of the chance occurrence of multiple malignant neoplasms. Am J Cancer 1934; 21: 2309-2824.

[8] Suzuki T, Takahashi H, Yao K: Multiple primary malignancies in the head and neck: a clinical review of 121 patients. acta otolaryngol suppl 2002, 547:88-92.

[9] Warren S, Gates O: Multiple primary malignant tumors: A survey of the literature and a statistical study. Am J Cancer 1932, 16:1358-1414.

[10] Moertel CG: Multiple primary malignant neoplasms: historical perspectives. Cancer 1977, 40(4 Suppl):1786-1792.

[11] Alhilli F, Das NS. Cancer in the Arabian Gulf Kingdom of Bahrain (1952-2004). Bahrain Med Bull 2010; 32: 57-60.

[12] Otrock ZK, Mahfouz RAR, Salem ZM. Four Primary Tumors of Lung, Bladder, Prostate and Breast in a Male Patient. Southern Med J 2005; 98: 946-9.

[13] Changhao C, Mengwei W. A Clinicopathological Analysis of 22 Cases of Multiple Malignant Tumors. Chin Med Sci J 2002; 17: 124-6.
[14] Beisland C, Talleraas O, Bakke A. Multiple Primary Malignancies in Patients with Renal Cell Carcinoma; A National Population-Based Cohort Study. BJU Int 2006; 97: 698-702.

[15] Haraguchi S, Hioki M, Koizumi K. Characteristics of Multiple Primary Malignancies Associated with Lung Cancer by Gender. Respiration 2006; 570: 1-4.

[16] Hayat MJ, Howlander N, Reichman ME. Cancer Statistics, Trends, and Multiple Primary Cancer Analysis from the Surveillance, Epidemiology, and End Results (SEER) Program. Oncologist 2007; 12: $20-37$

[17] International Rules for Multiple Primary Cancers (ICD-0 3rd Ed).Working Group Report. Eur J Cancer Prev 2005; 14(4): 307-8.

[18] Mariotto AB, Rowland JH, Ries LAG. Multiple Cancer Prevalence: A Growing Challenge in LongTerm Survivorship. Cancer Epidemiol Biomarkers Prev 2007; 16: 566-71.

[19] Jefferson KP, Gillatt DA(2007). Hereditary urological cancer syndromes. Nat Clin Pract Urol 4: 218-26.

[20] Orhan O, Gerald F, Carmen A, Raymond M. Incidental detection of synchronous primary tumours during staging workup for prostate cancer, Swiss Med WKLY. 2010;140(15-16):233-236.

[21] Lee SH, Chang PL, Chen SM, Sun GH, Chen SM, Sun GH, Chen CL, Shen BY, Wu YS, Tsui KH. Synchronous primary carcinomas of the bladder and prostate Asian $\mathrm{J}$

Androl. 2006 May 8(3):357-9.

[22] Abe T, Hayashi T, Nakayama J, Kishikawa $H$, Sekii K, Yoshioka T, Itatani H, Kojima Y, Takahara S. A case of synchronous double primary cancers of prostate, and bladder in a hemodialysis patient: a case report. Hinyokika Kiyo. 2005 Oct; 51(10):689-93.

[23] Yoshino K, Asanuma F, Hanatani Y, Kumai K, Ishibiki K: Statistical studies on multiple primary cancers including gastric cancers. Gan No Rinsho 1984, 30(12 Suppl):1514-1523. 